TRANSACTIONS OF THE

AMERICAN MATHEMATICAL SOCIETY

Volume 356, Number 11, Pages 4323-4342

S 0002-9947(04)03619-0

Article electronically published on May 28, 2004

\title{
MULTI-POINT TAYLOR EXPANSIONS OF ANALYTIC FUNCTIONS
}

\author{
JOSÉ L. LÓPEZ AND NICO M. TEMME
}

\begin{abstract}
Taylor expansions of analytic functions are considered with respect to several points, allowing confluence of any of them. Cauchy-type formulas are given for coefficients and remainders in the expansions, and the regions of convergence are indicated. It is explained how these expansions can be used in deriving uniform asymptotic expansions of integrals. The method is also used for obtaining Laurent expansions in several points as well as TaylorLaurent expansions.
\end{abstract}

\section{INTRODUCTION}

In deriving uniform asymptotic expansions of a certain class of integrals one encounters the problem of expanding a function that is analytic in some domain $\Omega$ of the complex plane, in several points. The first mention of the use of such expansions in asymptotics is given in [1, where Airy-type expansions are derived for integrals having two nearby (or coalescing) saddle points. This reference does not give further details about two-point Taylor expansions, because the coefficients in the Airy-type asymptotic expansion are derived in a different way. Other mentions of the use of such expansions in asymptotics are given in 7] and [5]. In [7], two-point Taylor expansions are used with applications to Airy-type expansions of parabolic cylinder functions. In [5] we used two-point Taylor expansions to derive convergent expansions of Charlier, Laguerre and Jacobi polynomials in terms of Gamma, Hermite and Chebyshev polynomials respectively.

To demonstrate an application in asymptotics of multi-Taylor expansions we may consider contour integrals of the form

$$
I(\lambda ; \boldsymbol{\alpha})=\int_{\mathcal{C}} g(z) e^{-\lambda f(z, \boldsymbol{\alpha})} d z
$$

where $\boldsymbol{\alpha}$ is a vector of parameters, $\boldsymbol{\alpha}=\left(\alpha_{1}, \ldots, \alpha_{2}\right)$, and the phase function $f(z, \boldsymbol{\alpha})$ has $m$ saddle points $z_{1}, z_{2}, \ldots, z_{m}$. The asymptotic behaviour of these integrals for large values of $\lambda$ is determined by the saddle-point structure of the phase function [9], Chapter 7, Section 6. One method for obtaining an asymptotic expansion of

Received by the editors November 14, 2002.

2000 Mathematics Subject Classification. Primary 30B10, 30E20; Secondary 40 A30.

Key words and phrases. Multi-point Taylor expansions, Cauchy's theorem, analytic functions, multi-point Laurent expansions, uniform asymptotic expansions of integrals.

The first author thanks the saving bank Caja Rural de Navarra for its financial support. He also acknowledges the scientific and financial support of CWI in Amsterdam.

The authors thank the referee for the comments on the first version of the paper. 
this integral for large values of $\lambda$ is based on expanding $g(z)$ at the saddle points of the phase function,

$$
g(z)=\sum_{n=0}^{\infty}\left[a_{0}+a_{1} z+\cdots+a_{m-1} z^{m-1}\right]\left(z-z_{1}\right)^{n}\left(z-z_{2}\right)^{n} \cdots\left(z-z_{m}\right)^{n}
$$

and substitution of this expansion into (1.1). When interchanging summation and integration, the result is a formal expansion in $m$ series in terms of functions related with the functions

$F_{n, k}(\lambda ; \boldsymbol{\alpha}) \equiv \int_{\mathcal{C}} z^{k}\left(z-z_{1}\right)^{n}\left(z-z_{2}\right)^{n} \cdots\left(z-z_{m}\right)^{n} e^{-\lambda f(z, \boldsymbol{\alpha})} d z, \quad k=1,2, \ldots, m-1$.

In [7], these functions $F_{n, k}(\lambda ; \boldsymbol{\alpha})$ are the Airy functions, whereas in [5] these functions are the Gamma function, or the Hermite or Chebyshev polynomials.

In a future paper we will use multi-point Taylor expansions in the asymptotic analysis of integrals arising in diffraction theory, such as the Bessel function integral (see [3] and [4])

$$
J(x, y)=\int_{0}^{\infty} t J_{0}(y t) e^{i\left(t^{4}+x t^{2}\right)} d t
$$

which is related to the Pearcey function

$$
\int_{-\infty}^{\infty} e^{i\left(\frac{1}{4} t^{4}+\frac{1}{2} x t^{2}+y t\right)} d t
$$

The Taylor-Laurent expansions will be used to study integrals with two saddle points and a pole of the integrand. Other applications in asymptotics include the study of Hermite-Padé approximations to the exponential function; in [2] integrals are considered with three saddle points.

In a recent paper [6] we have introduced the theory of two-point Taylor expansions, two-point Laurent expansions and two-point Taylor-Laurent expansions. The purpose of the present paper is to generalize that theory from 2 to $m$ points, $m \geq 2$. We give details on the region of convergence and on representations in terms of Cauchy-type integrals of the coefficients and the remainders of the expansions. Earlier information on this type of expansions is given in [8], Chapters 3 and 8 . The theory of several-point Taylor expansions was already formulated in Chapter 3 of Walsh's book, although in a different setting. Chapter 8 of 8 presents also a theory of rational approximation of analytic functions, but is different from the theory of multi-point Laurent and Taylor-Laurent expansions presented here. Whereas the multi-point polynomial approximation of Chapter 3 may be reformulated as a multi-point Taylor approximation, the rational approximation of Chapter 8 can not be written as a multi-point Laurent or Taylor-Laurent approximation. For more details, see Section 5.

\section{Multi-Point TAYlor EXPANSIOnS}

We consider the Taylor expansion of an analytic function $f(z)$ in several points and give information on the coefficients and the remainder in the expansion. In what follows empty sums and derivatives of negative order must be understood as zero and empty products as one. We will deal with the following set of points. 
Definition 2.1. We define the set

$$
S \equiv\left\{z_{1}, z_{1}, \ldots, z_{1} ; z_{2}, z_{2}, \ldots, z_{2}, \ldots ; z_{p}, z_{p}, \ldots, z_{p}\right\}
$$

of $m$ points consisting of $p$ different points $z_{1}, z_{2}, \ldots, z_{p}\left(z_{i} \neq z_{j}\right.$ if $\left.i \neq j\right)$, each $z_{j}$ repeated $m_{j}$ times: $m_{1}+m_{2}+\cdots+m_{p}=m$.

For clarity in the exposition, we first introduce the multi-point Taylor expansion for $m$ different points $z_{1}, z_{2}, \ldots, z_{m}\left(m=p, m_{j}=1\right)$ in Theorem 2.3. In Theorem 2.5 we assume that the points $z_{1}, z_{2}, \ldots, z_{m}$ may coalesce. We will need the following elementary lemma.

Lemma 2.2. Given $z, w \in \mathbb{C}$, take $m$ different points $z_{1}, z_{2}, \ldots, z_{m}$ in $\mathbb{C}$ and define

$$
H_{m}\left(w, z ; z_{1}, \ldots, z_{m}\right) \equiv \frac{\prod_{k=1}^{m}\left(w-z_{k}\right)-\prod_{k=1}^{m}\left(z-z_{k}\right)}{w-z} .
$$

Then

$$
H_{m}\left(w, z ; z_{1}, \ldots, z_{m}\right)=\sum_{j=1}^{m} \frac{\prod_{k=1, k \neq j}^{m}\left(w-z_{k}\right) \prod_{k=1, k \neq j}^{m}\left(z-z_{k}\right)}{\prod_{k=1, k \neq j}^{m}\left(z_{j}-z_{k}\right)} .
$$

Proof. The numerator of $H_{m}\left(w, z ; z_{1}, \ldots, z_{m}\right)$ is a polynomial of degree $m$ in the variable $w$ that vanishes at $w=z$. Therefore, $H_{m}\left(w, z ; z_{1}, \ldots, z_{m}\right)$ is a polynomial of degree $m-1$ in the variable $w$. Let $P_{m}\left(w, z ; z_{1}, \ldots, z_{m}\right)$ denote the function on the right-hand side of (2.3), which is also a polynomial of degree $m-1$ in the variable $w$. Moreover,

$$
H_{m}\left(z_{s}, z ; z_{1}, \ldots, z_{m}\right)=\prod_{k=1, k \neq s}^{m}\left(z-z_{k}\right)=P_{m}\left(z_{s}, z ; z_{1}, \ldots, z_{m}\right)
$$

for $s=1,2, \ldots, m$. Hence,

$$
H_{m}\left(w, z ; z_{1}, \ldots, z_{m}\right)=P_{m}\left(w, z ; z_{1}, \ldots, z_{m}\right) .
$$

Theorem 2.3. Let $f(z)$ be an analytic function on an open set $\Omega \subset \mathbb{C}$ and $S \subset \Omega$ with $S$ consisting of $m$ different points $(m=p)$. Then, $f(z)$ has the multi-point Taylor expansion

$$
f(z)=\sum_{n=0}^{N-1} q_{n, m}(z) \prod_{k=1}^{m}\left(z-z_{k}\right)^{n}+r_{N}(z)
$$

where $q_{n, m}(z)$ is the polynomial of degree $m-1$,

$$
q_{n, m}(z) \equiv \sum_{j=1}^{m} a_{n, j} \frac{\prod_{k=1, k \neq j}^{m}\left(z-z_{k}\right)}{\prod_{k=1, k \neq j}^{m}\left(z_{j}-z_{k}\right)},
$$

and the coefficients $a_{n, j}$ of this polynomial are given by the Cauchy integral

$$
a_{n, j} \equiv \frac{1}{2 \pi i} \int_{\mathcal{C}} \frac{f(w) d w}{\left(w-z_{j}\right) \prod_{k=1}^{m}\left(w-z_{k}\right)^{n}} .
$$

The remainder term $r_{N}(z)$ is given by the Cauchy integral

$$
r_{N}(z) \equiv \frac{1}{2 \pi i} \int_{\mathcal{C}} \frac{f(w) d w}{(w-z)\left[\prod_{k=1}^{m}\left(w-z_{k}\right)\right]^{N}}\left[\prod_{k=1}^{m}\left(z-z_{k}\right)\right]^{N} .
$$




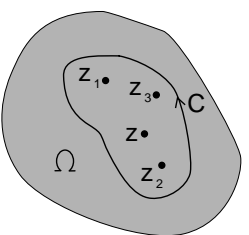

(a)

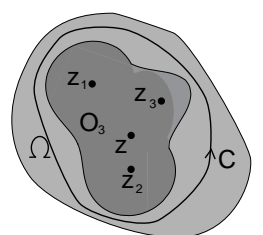

(b)

Figure 1. The case $m=3$. (a) Contour $\mathcal{C}$ in the integrals (2.8) and (2.9). (b) For $z \in O_{m}$, we can take a contour $\mathcal{C}$ in $\Omega$ that contains $O_{m}$ inside and, therefore, $\prod_{k=1}^{m}\left|z-z_{k}\right|<\prod_{k=1}^{m}\left|w-z_{k}\right|$ $\forall w \in \mathbb{C}$.

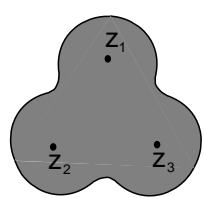

$r=r_{a}$

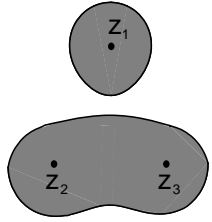

$r=r_{b}$

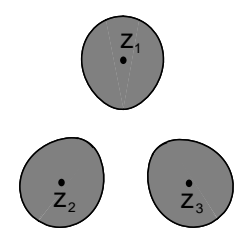

$r=r_{c}$

Figure 2. The shape of the "lemniscate domain" $O_{m}$ for $m=3$, which depends on the size of the parameter $r$ defined in (2.10). In these pictures $\left|z_{2}-z_{3}\right|<\left|z_{1}-z_{3}\right|,\left|z_{1}-z_{2}\right|$ and $r_{a}>r_{b}>r_{c}$.

The contour of integration $\mathcal{C}$ is a simple closed loop which encircles the points $z_{1}, z_{2}, \ldots, z_{m}$ (for $a_{n, j}$ ) and $z, z_{1}, z_{2}, \ldots, z_{m}$ (for $r_{N}(z)$ ) in the counterclockwise direction and is contained in $\Omega$ (see Figure 1(a)).

The expansion (2.6) is convergent for $z \in O_{m}$, where:

$$
O_{m} \equiv\left\{z \in \Omega, \prod_{k=1}^{m}\left|z-z_{k}\right|<r\right\}, \quad r \equiv \operatorname{Inf}_{w \in \mathbb{C} \backslash \Omega}\left\{\prod_{k=1}^{m}\left|w-z_{k}\right|\right\} .
$$

That is, (2.6) is convergent for $z$ inside the lemniscate $\prod_{k=1}^{m}\left|z-z_{k}\right|=r$ (see Figure 2; if $m=1$ this domain is a disk; if $m=2$ this domain is bounded by a Cassini oval). In particular, if $f(z)$ is an entire function $(\Omega=\mathbb{C})$, then the expansion (2.6) converges $\forall z \in \mathbb{C}$.

Proof. By Cauchy's theorem,

$$
f(z)=\frac{1}{2 \pi i} \int_{\mathcal{C}} \frac{f(w) d w}{w-z},
$$

where $\mathcal{C}$ is the contour defined above (Figure 1(a)). We write

$$
\frac{1}{w-z}=\frac{H_{m}\left(w, z ; z_{1}, \ldots, z_{m}\right)}{\prod_{k=1}^{m}\left(w-z_{k}\right)} \frac{1}{1-u},
$$

where $H_{m}\left(w, z ; z_{1}, \ldots, z_{m}\right)$ is given in (2.2) and

$$
u \equiv \frac{\prod_{k=1}^{m}\left(z-z_{k}\right)}{\prod_{k=1}^{m}\left(w-z_{k}\right)} .
$$


Now we use Lemma 2.2 and introduce the right-hand side of (2.3) and the expansion

$$
\frac{1}{1-u}=\sum_{n=0}^{N-1} u^{n}+\frac{u^{N}}{1-u}
$$

in (2.12) and this in (2.11). After straightforward calculations we obtain formulas (2.6) -(2.9)

For any $z \in O_{m}$, we can take a contour $\mathcal{C}$ in $\Omega$ such that

$$
\prod_{k=1}^{m}\left|z-z_{k}\right|<\prod_{k=1}^{m}\left|w-z_{k}\right|, \quad \forall w \in \mathbb{C}
$$

(see Figure 1(b)). On this contour $|f(w)|$ is bounded by some constant $C:|f(w)| \leq$ $C$. Introducing these two bounds in (2.9) we see that $\lim _{N \rightarrow \infty} r_{N}(z)=0$ and the proof follows.

We need the following lemma to consider the case of coalescing points in the set $S$.

Lemma 2.4. Given $z, w \in \mathbb{C}$, take $m$ different points $z_{1}, z_{2}, \ldots, z_{m}$ in $\mathbb{C}$, all different from $w$ too. Let those $m$ points coalesce at $z_{m}$, say. Then

$$
\lim _{z_{1}, z_{2}, \ldots, z_{m-1} \rightarrow z_{m}} \sum_{j=1}^{m} \frac{\prod_{k=1, k \neq j}^{m}\left(z-z_{k}\right)}{\left(w-z_{j}\right) \prod_{k=1, k \neq j}^{m}\left(z_{j}-z_{k}\right)}=\sum_{j=0}^{m-1} \frac{\left(z-z_{m}\right)^{j}}{\left(w-z_{m}\right)^{j+1}} .
$$

Proof. We first note that the identity

$$
\sum_{j=1}^{n} \prod_{l=1}^{j-1}\left(z_{n}-z_{l}\right) \prod_{l=j+1}^{n}\left(z_{1}-z_{l}\right)=0
$$

holds for any set of points $z_{1}, z_{2}, \ldots, z_{n}, n>1$. It may be checked in the following way: we take the first two terms of the sum, which gives

$$
\left(z_{n}-z_{2}\right)\left(z_{1}-z_{3}\right)\left(z_{1}-z_{4}\right) \cdots\left(z_{1}-z_{n-1}\right)\left(z_{1}-z_{n}\right) .
$$

Next we add to this the third term of the sum, which gives

$$
\left(z_{n}-z_{2}\right)\left(z_{n}-z_{3}\right)\left(z_{1}-z_{4}\right) \cdots\left(z_{1}-z_{n-1}\right)\left(z_{1}-z_{n}\right) .
$$

We continue this process until we add the $n$-1-th term of the sum, obtaining

$$
\left(z_{n}-z_{2}\right)\left(z_{n}-z_{3}\right)\left(z_{n}-z_{4}\right) \cdots\left(z_{n}-z_{n-1}\right)\left(z_{1}-z_{n}\right) \text {. }
$$

But this is just the last term of the sum with opposite sign.

Using the above identity we have

$$
\prod_{l=1}^{k-1}\left(z_{s}-z_{l}\right)\left[\sum_{j=k}^{s} \prod_{l=k}^{j-1}\left(z_{s}-z_{l}\right) \prod_{l=j+1}^{s}\left(z_{k}-z_{l}\right)\right] \prod_{l=s+1}^{m}\left(z_{k}-z_{l}\right)=0
$$

for any $s=1,2,3, \ldots, m$ and any $k=1,2,3, \ldots, m$ with $k \neq s$. Then

$$
\sum_{j=k}^{s} \prod_{l=1}^{j-1}\left(z_{s}-z_{l}\right) \prod_{l=j+1}^{m}\left(z_{k}-z_{l}\right)=\sum_{j=1}^{m} \prod_{l=1}^{j-1}\left(z_{s}-z_{l}\right) \prod_{l=j+1}^{m}\left(z_{k}-z_{l}\right)=0
$$

for any $s, k=1,2,3, \ldots, m$ with $k \neq s$. 
Now, for every $s=1,2,3, \ldots, m$, we define the following polynomials of degree $m-1$ in the variable $z$ :

$$
R_{s}(z) \equiv \prod_{l=1, l \neq s}^{m}\left(z-z_{l}\right), \quad S_{s}(z) \equiv \sum_{j=1}^{m} \prod_{l=1}^{j-1}\left(z-z_{l}\right) \prod_{l=j+1}^{m}\left(z_{s}-z_{l}\right) .
$$

The zeros of $R_{s}(z)$ are $z_{k}$ for $k=1,2,3, \ldots, m, k \neq s$ and from (2.22),$S_{s}\left(z_{k}\right)=0$ for $k=1,2,3, \ldots, m, k \neq s$. Moreover, the leading coefficient of $R_{s}(z)$ and that of $S_{s}(z)$ coincide. Therefore, $R_{s}(z)=S_{s}(z)$ for $s=1,2,3, \ldots, m$.

Finally, define the following polynomials of degree $m-1$ in the variable $w$ :

$$
\begin{gathered}
P(w, z) \equiv \sum_{j=1}^{m} \frac{\prod_{k=1, k \neq j}^{m}\left(z-z_{k}\right) \prod_{k=1, k \neq j}^{m}\left(w-z_{k}\right)}{\prod_{k=1, k \neq j}^{m}\left(z_{j}-z_{k}\right)}, \\
Q(w, z) \equiv \sum_{j=1}^{m} \prod_{k=1}^{j-1}\left(z-z_{k}\right) \prod_{k=j+1}^{m}\left(w-z_{k}\right) .
\end{gathered}
$$

For every $s=1,2,3, \ldots, m$ we have $P\left(z_{s}, z\right)=R_{s}(z)$ and $Q\left(z_{s}, z\right)=S_{s}(z)$. But $R_{s}(z)=S_{s}(z)$ and therefore $P(w, z)=Q(w, z)$. Then,

$$
\begin{aligned}
\sum_{j=1}^{m} \frac{\prod_{k=1, k \neq j}^{m}\left(z-z_{k}\right)}{\left(w-z_{j}\right) \prod_{k=1, k \neq j}^{m}\left(z_{j}-z_{k}\right)} & =\frac{P(w, z)}{\prod_{k=1}^{m}\left(w-z_{k}\right)} \\
& =\frac{Q(w, z)}{\prod_{k=1}^{m}\left(w-z_{k}\right)}=\sum_{j=1}^{m} \frac{\prod_{k=1}^{j-1}\left(z-z_{k}\right)}{\prod_{k=1}^{j}\left(w-z_{k}\right)} .
\end{aligned}
$$

Taking the limits $z_{1}, z_{2}, \ldots, z_{m-1} \rightarrow z_{m}$ on the left- and right-hand sides of these equalities, we obtain the desired result.

Theorem 2.5. Let $f(z)$ be an analytic function on an open set $\Omega \subset \mathbb{C}$ and $S \subset \Omega$. Then, $f(z)$ has the multi-point Taylor expansion

$$
f(z)=\sum_{n=0}^{N-1} q_{n, m}(z) \prod_{k=1}^{p}\left(z-z_{k}\right)^{n m_{k}}+r_{N}(z),
$$

where $q_{n, m}(z)$ is the polynomial of degree $m-1$,

$$
q_{n, m}(z) \equiv \sum_{j=1}^{p} \frac{\prod_{k=1, k \neq j}^{p}\left(z-z_{k}\right)^{m_{k}}}{\prod_{k=1, k \neq j}^{p}\left(z_{j}-z_{k}\right)^{m_{k}}} \sum_{l=0}^{m_{j}-1} a_{n, j, l}\left(z-z_{j}\right)^{l},
$$

and the coefficients $a_{n, j, l}$ of this polynomial are given by the Cauchy integral

$$
a_{n, j, l} \equiv \frac{1}{2 \pi i} \int_{\mathcal{C}} \frac{f(w) d w}{\left(w-z_{j}\right)^{l+1} \prod_{k=1}^{p}\left(w-z_{k}\right)^{n m_{k}}} .
$$

The remainder term $r_{N}(z)$ is given by the Cauchy integral

$$
r_{N}(z) \equiv \frac{1}{2 \pi i} \int_{\mathcal{C}} \frac{f(w) d w}{(w-z)\left[\prod_{k=1}^{p}\left(w-z_{k}\right)^{m_{k}}\right]^{N}}\left[\prod_{k=1}^{p}\left(z-z_{k}\right)^{m_{k}}\right]^{N} .
$$

The contour of integration $\mathcal{C}$ is a simple closed loop which encircles the points $z_{1}, z_{2}, \ldots, z_{p}\left(\right.$ for $\left.a_{n, j, l}\right)$ and $z, z_{1}, z_{2}, \ldots, z_{p}\left(\right.$ for $\left.r_{N}(z)\right)$ in the counterclockwise direction and is contained in $\Omega$ (see Figure 1(a)). 
The expansion (2.27) is convergent for $z \in O_{p}$ :

$$
O_{p} \equiv\left\{z \in \Omega, \prod_{k=1}^{p}\left|z-z_{k}\right|^{m_{k}}<r\right\}, \quad r \equiv \operatorname{Inf}_{w \in \mathbb{C} \backslash \Omega}\left\{\prod_{k=1}^{p}\left|w-z_{k}\right|^{m_{k}}\right\}
$$

that is, inside the lemniscate $\prod_{k=1}^{p}\left|z-z_{k}\right|^{m_{k}}=r$. In particular, if $f(z)$ is an entire function $(\Omega=\mathbb{C})$, then the expansion (2.27) converges $\forall z \in \mathbb{C}$.

Proof. If all the points in $S$ are different, we have from (2.7) and (2.8)

$$
q_{n, m}(z)=\frac{1}{2 \pi i} \int_{C} \frac{f(w) d w}{\prod_{k=1}^{m}\left(w-z_{k}\right)^{n}} \sum_{j=1}^{m} \frac{\prod_{k=1, k \neq j}^{m}\left(z-z_{k}\right)}{\left(w-z_{j}\right) \prod_{k=1, k \neq j}^{m}\left(z_{j}-z_{k}\right)} .
$$

This last sum may be also decomposed in the form

$$
\begin{aligned}
\sum_{j=1}^{m_{1}} & \frac{\prod_{k=1, k \neq j}^{m}\left(z-z_{k}\right)}{\left(w-z_{j}\right) \prod_{k=1, k \neq j}^{m}\left(z_{j}-z_{k}\right)}+\sum_{j=m_{1}+1}^{m_{2}} \frac{\prod_{k=1, k \neq j}^{m}\left(z-z_{k}\right)}{\left(w-z_{j}\right) \prod_{k=1, k \neq j}^{m}\left(z_{j}-z_{k}\right)} \\
& +\cdots+\sum_{j=m_{p-1}+1}^{m} \frac{\prod_{k=1, k \neq j}^{m}\left(z-z_{k}\right)}{\left(w-z_{j}\right) \prod_{k=1, k \neq j}^{m}\left(z_{j}-z_{k}\right)} .
\end{aligned}
$$

Now let the first $m_{1}$ points coalesce to $z_{1}$, the second $m_{2}$ points coalesce to $z_{2}$, and so on, and apply Lemma 2.4 to every one of the $p$ sums above to obtain (2.27), (2.28) and (2.29). Equation (2.30) follows from (2.9). The proof of the convergence of (2.27) in the region $O_{p}$ is a straightforward generalization of the corresponding proof in Theorem 2.3 .

2.1. Explicit forms of the coefficients. Formula (2.29) is not appropriate for numerical computations. A more practical formula to compute the coefficients of the above multi-point Taylor expansion is given in the following proposition. First we have a definition:

Definition 2.6. Let $f(w)$ be analytic at $w$; then for $n=0,1,2, \ldots$ the differential operator $D_{w}^{n} f(w)$ is defined by

$$
D_{w}^{n} f(w)=\frac{1}{n !} \frac{d^{n}}{d w^{n}} f(w) .
$$

Proposition 2.7. The coefficients $a_{n, j, l}$, for $n=1,2,3, \ldots, j=1,2, \ldots, p, l=$ $0,1, \ldots, m_{j}-1$ in the expansion (2.27) are also given by the formula:

$$
\begin{aligned}
a_{n, j, l}= & \left.D_{w}^{n m_{j}+l}\left[\frac{f(w)}{\prod_{s=1, s \neq j}^{p}\left(w-z_{s}\right)^{n m_{s}}}\right]\right|_{w=z_{j}} \\
& +\left.\sum_{k=1, k \neq j}^{p} D_{w}^{n m_{k}-1}\left[\frac{f(w)}{\left(w-z_{j}\right)^{l+1} \prod_{s=1, s \neq k}^{p}\left(w-z_{s}\right)^{n m_{s}}}\right]\right|_{w=z_{k}} .
\end{aligned}
$$

Proof. We deform the contour of integration $\mathcal{C}$ in equation (2.29) to any contour of the form $\mathcal{C}_{1} \cup \mathcal{C}_{2} \cup \cdots \cup \mathcal{C}_{p}$, also contained in $\Omega$, where $\mathcal{C}_{k}, k=1,2, \ldots, p$, is a simple closed loop which encircles the point $z_{k}$ in the counterclockwise direction 


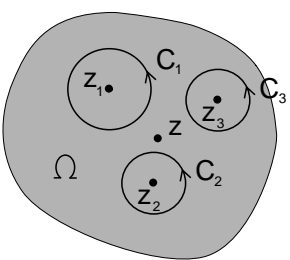

(a)

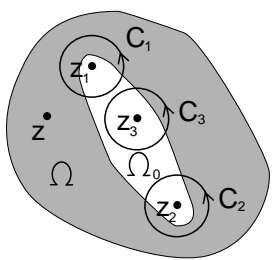

(b)

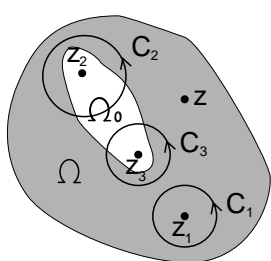

(c)

FiguRE 3. Integration contours $\mathcal{C}_{k}$ for $p=3$ and $q=1$.

(a) The function $\prod_{s=1, s \neq k}^{p}\left(w-z_{s}\right)^{-n m_{s}} f(w)$ is analytic inside $\mathcal{C}_{k}$ for $k=1,2, \ldots, p$.

(b) The functions

$\prod_{s=1, s \neq k}^{p}\left(w-z_{s}\right)^{-n m_{s}} g_{k}(w)$ and $\prod_{s=1, s \neq k}^{p}\left(w-z_{s}\right)^{(n+1) m_{s}} g_{k}(w)$ are analytic inside $\mathcal{C}_{k}$ for $k=1,2, \ldots, p$.

(c) The functions

$\prod_{s=1, s \neq k}^{p}\left(w-z_{s}\right)^{-n m_{s}} g_{k}(w)$,

$\prod_{s=1, s \neq k}^{q}\left(w-z_{s}\right)^{-n m_{s}} \prod_{s=q+1, s \neq k}^{p}\left(w-z_{s}\right)^{n m_{s}} g_{k}(w)$ and

$\prod_{s=1, s \neq k}^{q}\left(w-z_{s}\right)^{-(n+1) m_{s}} \prod_{s=q+1, s \neq k}^{p}\left(w-z_{s}\right)^{(n+1) m_{s}} g_{k}(w)$ are analytic inside $\mathcal{C}_{k}$ for $k=1,2, \ldots, p$.

and does not contain any other point $z_{j}, j=1,2, \ldots, p, j \neq k$ inside (see Figure 3 (a)). Then,

$$
\begin{aligned}
a_{n, j, l}= & \frac{1}{2 \pi i} \sum_{k=1, k \neq j}^{p} \int_{\mathcal{C}_{k}} \frac{f(w)}{\left(w-z_{j}\right)^{l+1} \prod_{s=1, s \neq k}^{p}\left(w-z_{s}\right)^{n m_{s}}} \frac{d w}{\left(w-z_{k}\right)^{n m_{k}}} \\
& +\frac{1}{2 \pi i} \int_{\mathcal{C}_{j}} \frac{f(w)}{\prod_{s=1, s \neq j}^{p}\left(w-z_{s}\right)^{n m_{s}}} \frac{d w}{\left(w-z_{j}\right)^{n m_{j}+l+1}},
\end{aligned}
$$

from which equation (2.35) follows.

2.2. Multi-point Taylor polynomials. In Theorem 2.3 we have assumed that the function $f(z)$ is analytic in $\Omega$. If $f(z)$ is not analytic in $\Omega$ but has a finite number of derivatives at $z_{1}, z_{2}, \ldots, z_{p}$, we can still define the multi-point Taylor polynomial of the function $f(z)$ at $z_{1}, z_{2}, \ldots, z_{p}$ in the following way:

Definition 2.8. Let $z$ be a real or complex variable. If $f(z)$ is $N m_{k}-1$ times differentiable at $z_{1}, z_{2}, \ldots, z_{p}$, we define the multi-point Taylor polynomial of degree $m N-1$ of $f(z)$ at the points of $S$ as

$$
P_{N}(z) \equiv \sum_{n=0}^{N-1} q_{n, m}(z) \prod_{k=1}^{p}\left(z-z_{k}\right)^{n m_{k}},
$$

where $q_{n, m}(z)$ is the polynomial of degree $m-1$

$$
q_{n, m}(z) \equiv \sum_{j=1}^{p} \frac{\prod_{k=1, k \neq j}^{p}\left(z-z_{k}\right)^{m_{k}}}{\prod_{k=1, k \neq j}^{p}\left(z_{j}-z_{k}\right)^{m_{k}}} \sum_{l=0}^{m_{j}-1} a_{n, j, l}\left(z-z_{j}\right)^{l}
$$

and the coefficients $a_{n, j, l}$ are given in (2.35). 
Proposition 2.9. In the conditions of the above definition, define the remainder of the approximation of $f(z)$ by $P_{N}(z)$ at the points of $S$ as

$$
r_{N}(z) \equiv f(z)-P_{N}(z) .
$$

Then, (i) $r_{N}(z)=o\left(z-z_{k}\right)^{N m_{k}-1}$ as $z \rightarrow z_{k}, k=1,2, \ldots, p$. (ii) If $f(z)$ is $N m_{k}$ times differentiable at $z_{k}$ for some $k$, then $r_{N}(z)=O\left(z-z_{k}\right)^{N m_{k}}$ as $z \rightarrow z_{k}$.

Proof. The proof is trivial if $f(z)$ is analytic at every $z_{1}, z_{2}, \ldots, z_{p}$ by using (2.30). In any case, for a real or complex variable, the proof follows by using l'Hôpital's rule and (2.35).

Remark 2.10. Observe that the degree $m N-1$ Taylor polynomial of $f(z)$ at the points of $S$ is Hermite's interpolation polynomial of $f(z)$ at $z_{1}, z_{2}, \ldots, z_{p}$ with data $f\left(z_{k}\right), f^{\prime}\left(z_{k}\right), \ldots, f^{\left(N m_{k}-1\right)}\left(z_{k}\right), k=1,2, \ldots, p$.

\section{Multi-Point Laurent expansions}

In the standard theory of Taylor and Laurent expansions much analogy exists between the two types of expansions. For multi-point expansions, we have a similar resemblance in the representations of coefficients and remainders.

Theorem 3.1. Let $\Omega_{0}$ and $\Omega$ be a closed and an open set, respectively, of the complex plane, and $\Omega_{0} \subset \Omega \subset \mathbb{C}$. Let $f(z)$ be an analytic function on $\Omega \backslash \Omega_{0}$ and $z_{1}, z_{2}, \ldots, z_{p} \in \Omega_{0}$ (that is, $S \in \Omega_{0}$ ). Then, for any $z \in \Omega \backslash \Omega_{0}, f(z)$ has the multi-point Laurent expansion

$$
f(z)=\sum_{n=0}^{N-1} q_{n, m}(z) \prod_{k=1}^{p}\left(z-z_{k}\right)^{n m_{k}}+\sum_{n=0}^{N-1} t_{n, m}(z) \prod_{k=1}^{p}\left(z-z_{k}\right)^{-(n+1) m_{k}}+r_{N}(z)
$$

where $q_{n, m}(z)$ is the polynomial of degree $m-1$,

$$
q_{n, m}(z) \equiv \sum_{j=1}^{p} \frac{\prod_{k=1, k \neq j}^{p}\left(z-z_{k}\right)^{m_{k}}}{\prod_{k=1, k \neq j}^{p}\left(z_{j}-z_{k}\right)^{m_{k}}} \sum_{l=0}^{m_{j}-1} a_{n, j, l}\left(z-z_{j}\right)^{l},
$$

and the coefficients $a_{n, j, l}$ of this polynomial are given by the Cauchy integral

$$
a_{n, j, l} \equiv \frac{1}{2 \pi i} \int_{\Gamma_{1}} \frac{f(w) d w}{\left(w-z_{j}\right)^{l+1} \prod_{k=1}^{p}\left(w-z_{k}\right)^{n m_{k}}} .
$$

Also, $t_{n, m}(z)$ is the polynomial of degree $m-1$,

$$
t_{n, m}(z) \equiv \sum_{j=1}^{p} \frac{\prod_{k=1, k \neq j}^{p}\left(z-z_{k}\right)^{m_{k}}}{\prod_{k=1, k \neq j}^{p}\left(z_{j}-z_{k}\right)^{m_{k}}} \sum_{l=0}^{m_{j}-1} b_{n, j, l}\left(z-z_{j}\right)^{l},
$$

where the coefficients $b_{n, j, l}$ of this polynomial are given by the Cauchy integral

$$
b_{n, j, l} \equiv \frac{1}{2 \pi i} \int_{\Gamma_{2}} \prod_{k=1}^{p}\left(w-z_{k}\right)^{m_{k}(n+1)} \frac{f(w) d w}{\left(w-z_{j}\right)^{l+1}} .
$$




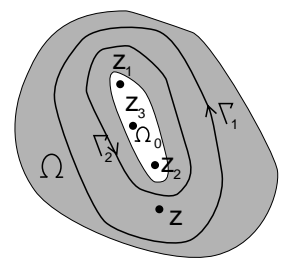

(a)

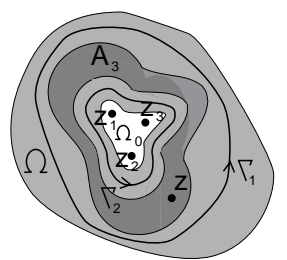

(b)

Figure 4 . The case $p=3$. (a) Contours $\Gamma_{1}$ and $\Gamma_{2}$ in the integrals (3.3), (3.5) and (3.6). (b) For $z \in A_{p}$, we can take a contour $\Gamma_{2}$ in $\Omega$ located between $\Omega_{0}$ and $A_{p}$ and a contour $\Gamma_{1}$ in $\Omega$ such that $A_{p}$ is inside this contour. Therefore, $\prod_{k=1}^{p}\left|z-z_{k}\right|^{m_{k}}<\prod_{k=1}^{p}\left|w-z_{k}\right|^{m_{k}}$ $\forall w \in \Gamma_{1}$ and $\prod_{k=1}^{p}\left|w-z_{k}\right|^{m_{k}}<\prod_{k=1}^{p}\left|z-z_{k}\right|^{m_{k}} \forall w \in \Gamma_{2}$.

The remainder term $r_{N}(z)$ is given by the Cauchy integrals

$$
\begin{aligned}
r_{N}(z) \equiv & \frac{1}{2 \pi i} \int_{\Gamma_{1}} \frac{f(w) d w}{(w-z) \prod_{k=1}^{p}\left(w-z_{k}\right)^{N m_{k}}} \prod_{k=1}^{p}\left(z-z_{k}\right)^{N m_{k}} \\
& -\frac{1}{2 \pi i} \int_{\Gamma_{2}} \prod_{k=1}^{p}\left(w-z_{k}\right)^{N m_{k}} \frac{f(w) d w}{w-z} \frac{1}{\prod_{k=1}^{p}\left(z-z_{k}\right)^{N m_{k}}} .
\end{aligned}
$$

In these integrals, the contours of integration $\Gamma_{1}$ and $\Gamma_{2}$ are simple closed loops contained in $\Omega \backslash \Omega_{0}$ which encircle the points $z_{1}, z_{2}, \ldots, z_{p}$ in the counterclockwise direction. Moreover, $z$ is not inside $\Gamma_{2}$, whereas $\Gamma_{1}$ encircles $\Gamma_{2}$ and the point $z$ (see Figure 4(a)).

The expansion (3.1) is convergent for $z$ inside the "lemniscate annulus" (see Figure 5)

$$
A_{p} \equiv\left\{z \in \Omega \backslash \Omega_{0}, \quad r_{2}<\prod_{k=1}^{p}\left|z-z_{k}\right|^{m_{k}}<r_{1}\right\}
$$

where

$$
r_{1} \equiv \operatorname{Inf}_{w \in \mathbb{C} \backslash \Omega}\left\{\prod_{k=1}^{p}\left|w-z_{k}\right|^{m_{k}}\right\}, \quad r_{2} \equiv \operatorname{Sup}_{w \in \Omega_{0}}\left\{\prod_{k=1}^{p}\left|w-z_{k}\right|^{m_{k}}\right\} .
$$

Proof. By Cauchy's theorem,

$$
f(z)=\frac{1}{2 \pi i} \int_{\Gamma_{1}} \frac{f(w) d w}{w-z}-\frac{1}{2 \pi i} \int_{\Gamma_{2}} \frac{f(w) d w}{w-z},
$$

where $\Gamma_{1}$ and $\Gamma_{2}$ are the contours defined above. First we assume that the $m$ points of the set $S$ are all distinct and later we will let the first $m_{1}$ points coalesce to $z_{1}$, the second $m_{2}$ points coalesce to $z_{2}$ and so on. We substitute (2.12)-(2.13) into the first integral above and

$$
\frac{1}{w-z}=-\frac{H_{m}\left(w, z ; z_{1}, \ldots, z_{m}\right)}{\prod_{k=1}^{m}\left(z-z_{k}\right)} \frac{1}{1-u}, \quad u \equiv \frac{\prod_{k=1}^{m}\left(w-z_{k}\right)}{\prod_{k=1}^{m}\left(z-z_{k}\right)},
$$

where $H_{m}\left(w, z ; z_{1}, \ldots, z_{m}\right)$ is defined in (2.2), into the second integral. Now we introduce the expansion (2.14) of the factor $(1-u)^{-1}$ in both integrals in (3.9). 


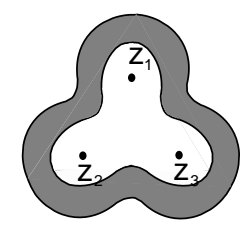

$\left(r_{1}^{a}, r_{2}^{a}\right)$
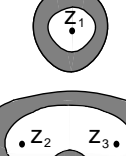

$\left(r_{1}^{b}, r_{2}^{b}\right)$

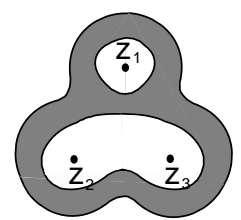

$\left(r_{1}^{a}, r_{2}^{b}\right)$

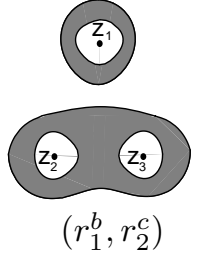

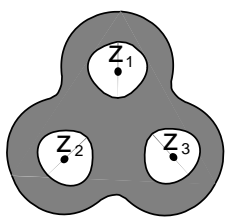

$\left(r_{1}^{a}, r_{2}^{c}\right)$

Figure 5. Shape of the "lemniscate annulus" $A_{p}$ for $p=3$, which depends on the relative size of the parameters $r_{1}$ and $r_{2}$ defined in (3.8). The different forms are labeled by $\left(r_{1}, r_{2}\right)$ with $r_{1}>r_{2}$. In these pictures $\left|z_{2}-z_{3}\right|<\left|z_{1}-z_{3}\right|,\left|z_{1}-z_{2}\right|$ and $r_{2}^{a}>r_{2}^{b}>r_{2}^{c}$.

Using (2.3) and after straightforward calculations we obtain

$$
f(z)=\sum_{n=0}^{N-1} q_{n, m}(z) \prod_{k=1}^{m}\left(z-z_{k}\right)^{n}+\sum_{n=0}^{N-1} t_{n, m}(z) \prod_{k=1}^{m}\left(z-z_{k}\right)^{-n-1}+r_{N}(z),
$$

where $q_{n, m}(z)$ is given by formulas (2.7) and (2.8) replacing the contour $\mathcal{C}$ by $\Gamma_{1}$. Also,

$t_{n, m}(z)=\sum_{j=1}^{m} b_{n, j} \frac{\prod_{k=1, k \neq j}^{m}\left(z-z_{k}\right)}{\prod_{k=1, k \neq j}^{m}\left(z_{j}-z_{k}\right)}, \quad b_{n, j} \equiv \frac{1}{2 \pi i} \int_{\Gamma_{2}} \prod_{k=1}^{m}\left(w-z_{k}\right)^{n+1} \frac{f(w) d w}{w-z_{j}}$,

and

$$
\begin{aligned}
r_{N}(z)= & \frac{1}{2 \pi i} \int_{\Gamma_{1}} \frac{f(w) d w}{(w-z) \prod_{k=1}^{m}\left(w-z_{k}\right)^{N}} \prod_{k=1}^{m}\left(z-z_{k}\right)^{N} \\
& -\frac{1}{2 \pi i} \int_{\Gamma_{2}} \prod_{k=1}^{m}\left(w-z_{k}\right)^{N} \frac{f(w) d w}{w-z} \frac{1}{\prod_{k=1}^{m}\left(z-z_{k}\right)^{N}} .
\end{aligned}
$$

Now we write

$$
t_{n, m}(z)=\frac{1}{2 \pi i} \int_{\Gamma_{2}} \prod_{k=1}^{m}\left(w-z_{k}\right)^{n+1} f(w) d w \sum_{j=1}^{m} \frac{\prod_{k=1, k \neq j}^{m}\left(z-z_{k}\right)}{\left(w-z_{j}\right) \prod_{k=1, k \neq j}^{m}\left(z_{j}-z_{k}\right)}
$$

and repeat the steps following (2.32) in the proof of Theorem 2.5 for $q_{n, m}(z)$ and $t_{n, m}(z)$. 
For any $z \in A_{p}$ we can take simple closed loops $\Gamma_{1}$ and $\Gamma_{2}$ in $\Omega \backslash \Omega_{0}$ such that (see Figure4(b))

$$
\prod_{k=1}^{p}\left|z-z_{k}\right|^{m_{k}}<\prod_{k=1}^{p}\left|w-z_{k}\right|^{m_{k}} \quad \forall w \in \Gamma_{1}
$$

and

$$
\prod_{k=1}^{p}\left|z-z_{k}\right|^{m_{k}}>\prod_{k=1}^{p}\left|w-z_{k}\right|^{m_{k}} \quad \forall w \in \Gamma_{2} .
$$

On these contours $|f(w)|$ is bounded by some constant $C:|f(w)| \leq C$. Introducing these bounds in (3.6) we see that $\lim _{N \rightarrow \infty} r_{N}(z)=0$ and the proof follows.

If the only singularities of $f(z)$ inside $\Omega_{0}$ are just poles at $z_{1}, z_{2}, \ldots, z_{p}$, then alternative formulas of (3.3) and (3.5) for computing the coefficients of the above multi-point Laurent expansion are given in the following proposition.

Proposition 3.2. Suppose that $g_{k}(z) \equiv\left(z-z_{k}\right)^{\rho_{k}} f(z), k=1,2, \ldots, p$, are analytic functions in $\Omega$ for certain $\rho_{1}, \rho_{2}, \ldots, \rho_{k} \in \mathbb{N}$. Then, for $n=0,1,2, \ldots$, the coefficients $a_{n, j, l}$ and $b_{n, j, l}$ in expansion (3.1) are also given by the formulas:

$$
\begin{aligned}
a_{n, j, l}= & \left.\sum_{k=1, k \neq j}^{p} D_{w}^{n m_{k}+\rho_{k}-1}\left[\frac{g_{k}(w)}{\left(w-z_{j}\right)^{l+1} \prod_{s=1, s \neq k}^{p}\left(w-z_{s}\right)^{n m_{s}}}\right]\right|_{w=z_{k}} \\
& +\left.D_{w}^{n m_{j}+\rho_{j}+l}\left[\frac{g_{j}(w)}{\prod_{s=1, s \neq j}^{p}\left(w-z_{s}\right)^{n m_{s}}}\right]\right|_{w=z_{j}}
\end{aligned}
$$

and

$$
\begin{aligned}
b_{n, j, l}= & \left.\sum_{k=1, k \neq j}^{p} D_{w}^{\rho_{k}-(n+1) m_{k}-1}\left[\frac{g_{k}(w)}{\left(w-z_{j}\right)^{l+1}} \prod_{s=1, s \neq k}^{p}\left(w-z_{s}\right)^{(n+1) m_{s}}\right]\right|_{w=z_{k}} \\
& +\left.D_{w}^{\rho_{j}-(n+1) m_{j}+l}\left[g_{j}(w) \prod_{s=1, s \neq j}^{p}\left(w-z_{s}\right)^{(n+1) m_{s}}\right]\right|_{w=z_{j}} .
\end{aligned}
$$

Proof. We deform both contours $\Gamma_{1}$ and $\Gamma_{2}$ of equations (3.3) and (3.5), respectively, to any contour of the form $\mathcal{C}_{1} \cup \mathcal{C}_{2} \cup \cdots \cup \mathcal{C}_{p}$ contained in $\Omega$, where $\mathcal{C}_{k}, k=1,2, \ldots, p$, is a simple closed loop which encircles the point $z_{k}$ in the counterclockwise direction and does not contain the point $z_{j}, j=1,2, \ldots, p, j \neq k$, inside (see Figure 3(b)). Then,

$$
\begin{aligned}
a_{n, j, l}= & \frac{1}{2 \pi i} \sum_{k=1, k \neq j}^{p} \int_{\mathcal{C}_{k}} \frac{g_{k}(w)}{\left(w-z_{j}\right)^{l+1} \prod_{s=1, s \neq k}^{p}\left(w-z_{s}\right)^{n m_{s}}} \frac{d w}{\left(w-z_{k}\right)^{n m_{k}+\rho_{k}}} \\
& +\frac{1}{2 \pi i} \int_{\mathcal{C}_{j}} \frac{g_{j}(w)}{\prod_{s=1, s \neq j}^{p}\left(w-z_{s}\right)^{n m_{s}}} \frac{d w}{\left(w-z_{j}\right)^{n m_{j}+\rho_{j}+l+1}}
\end{aligned}
$$


and

$$
\begin{aligned}
b_{n, j, l}= & \frac{1}{2 \pi i} \sum_{k=1, k \neq j}^{p} \int_{\mathcal{C}_{k}} \frac{\prod_{s=1, s \neq k}^{p}\left(w-z_{s}\right)^{(n+1) m_{s}}}{\left(w-z_{j}\right)^{l+1}} \frac{g_{k}(w) d w}{\left(w-z_{k}\right)^{\rho_{k}-(n+1) m_{k}}} \\
& +\frac{1}{2 \pi i} \int_{\mathcal{C}_{j}} \prod_{s=1, s \neq j}^{p}\left(w-z_{s}\right)^{(n+1) m_{s}} \frac{g_{j}(w) d w}{\left(w-z_{j}\right)^{\rho_{j}-(n+1) m_{j}+l+1}} .
\end{aligned}
$$

From this, equations (3.17) and (3.18) follow.

Remark 3.3. Let $z$ be a real or complex variable. Suppose $g_{k}(z) \equiv\left(z-z_{k}\right)^{\rho_{k}} f(z)$ is $\rho_{k}-1$ times differentiable at every $z_{k}$ in $S$ for some $\rho_{k} \in \mathbb{N}, k=1,2, \ldots, p$. Define

$$
g(z) \equiv f(z)-\sum_{n=0}^{M} t_{n, m}(z) \prod_{k=1}^{p}\left(z-z_{k}\right)^{-(n+1) m_{k}},
$$

where $M \equiv\left\lfloor\operatorname{Max}\left\{\left(\rho_{1}-1\right) / m_{1},\left(\rho_{2}-1\right) / m_{2}, \ldots,\left(\rho_{p}-1\right) / m_{p}\right\}\right\rfloor$ and $t_{n, m}(z)$ is the polynomial defined in (3.4) and (3.18). Then, the thesis of Proposition [2.9] holds for $f(z)$ replaced by $g(z)$. Moreover, if $\prod_{k=1}^{p}\left(z-z_{k}\right)^{\rho_{k}} f(z)$ is an analytic function in $\Omega$, then the thesis of Theorem 2.5 applies to $g(z)$.

\section{Multi-Point Taylor-Laurent expansions}

For multi-point expansions we have the possibility (that we do not have in the standard theory) of expanding in Taylor series at some points and in Laurent series at other points.

Theorem 4.1. Let $\Omega_{0}$ and $\Omega$ be closed and open sets, respectively, of the complex plane, and $\Omega_{0} \subset \Omega \subset \mathbb{C}$. Let $f(z)$ be an analytic function on $\Omega \backslash \Omega_{0}, z_{1}, z_{2}, \ldots, z_{q} \in$ $\Omega \backslash \Omega_{0}$ and $z_{q+1}, z_{q+2}, \ldots, z_{p} \in \Omega_{0}$ (q points are in $\Omega \backslash \Omega_{0}$ and $p-q$ points are in $\Omega_{0}$ ). Write $s \equiv m_{1}+m_{2}+\cdots+m_{q}$. Then, for $z \in \Omega \backslash \Omega_{0}, f(z)$ has the Taylor-Laurent expansion

$$
\begin{aligned}
f(z)= & \sum_{n=0}^{N-1} q_{n, m}(z) \prod_{k=1}^{p}\left(z-z_{k}\right)^{n m_{k}}+\sum_{n=0}^{N-1} t_{n, m}^{(1)}(z) \frac{\prod_{k=1}^{q}\left(z-z_{k}\right)^{n m_{k}}}{\prod_{k=q+1}^{p}\left(z-z_{k}\right)^{n m_{k}}} \\
& +\sum_{n=0}^{N-1} t_{n, m}^{(2)}(z) \frac{\prod_{k=1}^{q}\left(z-z_{k}\right)^{(n+1) m_{k}}}{\prod_{k=q+1}^{p}\left(z-z_{k}\right)^{(n+1) m_{k}}}+r_{N}(z),
\end{aligned}
$$

where $q_{n, m}(z)$ is the polynomial of degree $m-1$,

$$
q_{n, m}(z) \equiv \sum_{j=1}^{p} \frac{\prod_{k=1, k \neq j}^{p}\left(z-z_{k}\right)^{m_{k}}}{\prod_{k=1, k \neq j}^{p}\left(z_{j}-z_{k}\right)^{m_{k}}} \sum_{l=0}^{m_{j}-1} a_{n, j, l}\left(z-z_{j}\right)^{l},
$$

and the coefficients $a_{n, j, l}$ of this polynomial are given by the Cauchy integral

$$
a_{n, j, l} \equiv \frac{1}{2 \pi i} \int_{\Gamma_{1}} \frac{f(w) d w}{\left(w-z_{j}\right)^{l+1} \prod_{k=1}^{p}\left(w-z_{k}\right)^{n m_{k}}} .
$$

Also, $t_{n, m}^{(1)}(z)$ and $t_{n, m}^{(2)}(z)$ are the following polynomials of degrees $s-1$ and $m-s-1$, respectively:

$$
t_{n, m}^{(1)}(z) \equiv-\sum_{j=1}^{q} \frac{\prod_{k=1, k \neq j}^{q}\left(z-z_{k}\right)^{m_{k}}}{\prod_{k=1, k \neq j}^{q}\left(z_{j}-z_{k}\right)^{m_{k}}} \sum_{l=0}^{m_{j}-1} b_{n, j, l}\left(z-z_{j}\right)^{l},
$$


where the coefficients $b_{n, j, l}$ of this polynomial are given by the Cauchy integral

$$
b_{n, j, l} \equiv \frac{1}{2 \pi i} \int_{\Gamma_{2}} \frac{\prod_{k=q+1}^{p}\left(w-z_{k}\right)^{n m_{k}}}{\prod_{k=1}^{q}\left(w-z_{k}\right)^{n m_{k}}} \frac{f(w) d w}{\left(w-z_{j}\right)^{l+1}},
$$

and

$$
t_{n, m}^{(2)}(z) \equiv \sum_{j=q+1}^{p} \frac{\prod_{k=q+1, k \neq j}^{p}\left(z-z_{k}\right)^{m_{k}}}{\prod_{k=q+1, k \neq j}^{p}\left(z_{j}-z_{k}\right)^{m_{k}}} \sum_{l=0}^{m_{j}-1} c_{n, j, l}\left(z-z_{j}\right)^{l},
$$

where the coefficients $c_{n, j, l}$ of this polynomial are given by the Cauchy integral

$$
c_{n, j, l} \equiv \frac{1}{2 \pi i} \int_{\Gamma_{2}} \frac{\prod_{k=q+1}^{p}\left(w-z_{k}\right)^{(n+1) m_{k}}}{\prod_{k=1}^{q}\left(w-z_{k}\right)^{(n+1) m_{k}}} \frac{f(w) d w}{\left(w-z_{j}\right)^{l+1}} .
$$

The remainder term $r_{N}(z)$ is given by the Cauchy integrals

$$
\begin{aligned}
r_{N}(z) \equiv & \frac{1}{2 \pi i} \int_{\Gamma_{1}} \frac{f(w) d w}{(w-z) \prod_{k=1}^{p}\left(w-z_{k}\right)^{N m_{k}}} \prod_{k=1}^{p}\left(z-z_{k}\right)^{N m_{k}} \\
& -\frac{1}{2 \pi i} \int_{\Gamma_{2}} \frac{\prod_{k=q+1}^{p}\left(w-z_{k}\right)^{N m_{k}}}{\prod_{k=1}^{q}\left(w-z_{k}\right)^{N m_{k}}} \frac{f(w) d w}{w-z} \frac{\prod_{k=1}^{q}\left(z-z_{k}\right)^{N m_{k}}}{\prod_{k=q+1}^{p}\left(z-z_{k}\right)^{N m_{k}}} .
\end{aligned}
$$

In these integrals, the contours of integration $\Gamma_{1}$ and $\Gamma_{2}$ are simple closed loops contained in $\Omega \backslash \Omega_{0}$ which encircle $\Omega_{0}$ in the counterclockwise direction. Moreover, the points $z$ and $z_{1}, z_{2}, \ldots, z_{q}$ are not inside $\Gamma_{2}$, whereas $\Gamma_{1}$ encircles $\Gamma_{2}$ and the points $z$ and $z_{1}, z_{2}, \ldots, z_{q}$ (see Figure $6(a)$ ).

The expansion (4.1) is convergent in the region (Figure 7)

$$
\begin{aligned}
D_{q, p} \equiv\left\{z \in \Omega \backslash \Omega_{0},\right. & \prod_{k=1}^{p}\left|\left(z-z_{k}\right)\right|^{m_{k}}<r_{1}, \\
& \left.\prod_{k=1}^{q}\left|\left(z-z_{k}\right)\right|^{m_{k}}<r_{2} \prod_{k=q+1}^{p}\left|\left(z-z_{k}\right)\right|^{m_{k}}\right\}
\end{aligned}
$$

where $r_{1} \equiv \operatorname{Inf}_{w \in \mathbb{C} \backslash \Omega}\left\{\prod_{k=1}^{p}\left|\left(w-z_{k}\right)\right|^{m_{k}}\right\}$ and

$$
r_{2} \equiv \operatorname{Inf}_{w \in \Omega_{0}}\left\{\prod_{k=1}^{q}\left|\left(w-z_{k}\right)\right|^{m_{k}} \prod_{k=q+1}^{p}\left|\left(w-z_{k}\right)^{-1}\right|^{m_{k}}\right\} .
$$

Proof. By Cauchy's theorem,

$$
f(z)=\frac{1}{2 \pi i} \int_{\Gamma_{1}} \frac{f(w) d w}{w-z}-\frac{1}{2 \pi i} \int_{\Gamma_{2}} \frac{f(w) d w}{w-z},
$$

where $\Gamma_{1}$ and $\Gamma_{2}$ are the contours defined above.

First we assume that the $m$ points of the set $S$ are all distinct. Later we will let the first $m_{1}$ points coalesce to $z_{1}$, the second $m_{2}$ points to $z_{2}$, and so on. We substitute (2.12) $-(2.13)$ into the first integral above and

$$
\frac{1}{w-z}=\frac{F_{m}\left(w, z ; z_{1}, \ldots, z_{m}\right)}{\prod_{k=1}^{s}\left(w-z_{k}\right) \prod_{k=s+1}^{m}\left(z-z_{k}\right)} \frac{1}{1-u},
$$




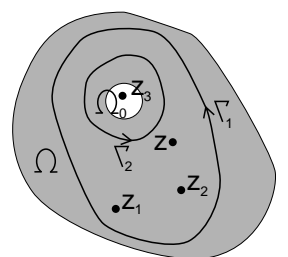

(a)

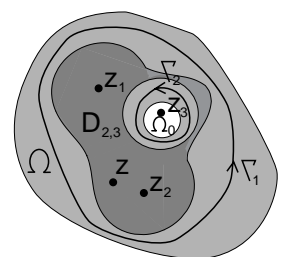

(b)

Figure 6 . The case $q=2, p=3$. (a) Contours $\Gamma_{1}$ and $\Gamma_{2}$ in the integrals (4.3), (4.5), (4.7) and (4.8). (b) For $z \in D_{q, p}$, we can take a contour $\Gamma_{2}$ located between $\Omega_{0}$ and $D_{q, p}$ and a contour $\Gamma_{1}$ in $\Omega$ with $D_{q, p}$ inside this contour. Therefore, $\prod_{k=1}^{p}\left|z-z_{k}\right|^{m_{k}}<$ $\prod_{k=1}^{p}\left|w-z_{k}\right|^{m_{k}} \forall w \in \Gamma_{1}$ and $\prod_{k=1}^{q}\left|z-z_{k}\right|^{m_{k}} \prod_{k=q+1}^{p}\left|w-z_{k}\right|^{m_{k}}<$ $\prod_{k=1}^{q}\left|w-z_{k}\right|^{m_{k}} \prod_{k=q+1}^{p}\left|z-z_{k}\right|^{m_{k}} \forall w \in \Gamma_{2}$.

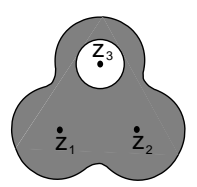

$\left(r_{1}^{a}, r_{2}^{a}\right)$

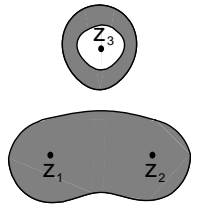

$\left(r_{1}^{b}, r_{2}^{a}\right)$

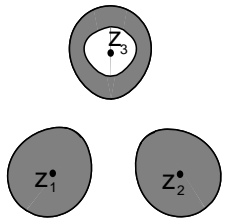

$\left(r_{1}^{c}, r_{2}^{a}\right)$

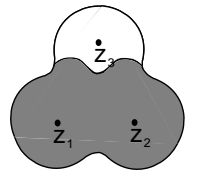

$\left(r_{1}^{a}, r_{2}^{b}\right)$

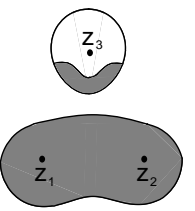

$\left(r_{1}^{b}, r_{2}^{b}\right)$

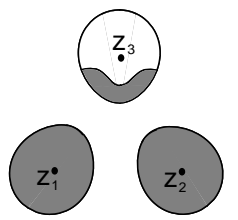

$\left(r_{1}^{c}, r_{2}^{b}\right)$

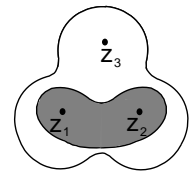

$\left(r_{1}^{a}, r_{2}^{c}\right)$
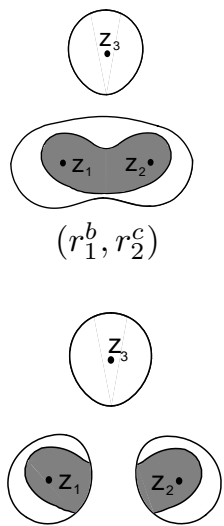

$\left(r_{1}^{c}, r_{2}^{c}\right)$
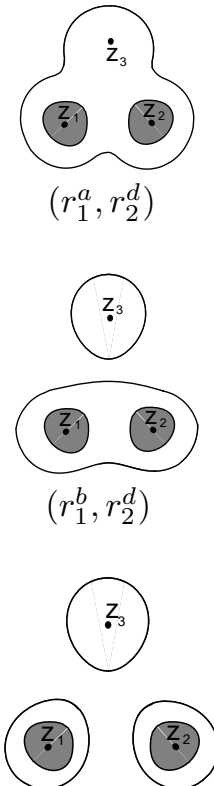

$\left(r_{1}^{c}, r_{2}^{d}\right)$

Figure 7 . The region $D_{q, p}$ defined in Theorem 4.1 is given by $D_{q, p}=O_{p} \cap B_{q, p}$, where $O_{p}$ is the "lemniscate domain" of foci $z_{1}, \ldots, z_{p}$ and parameter $r_{1}$. Also, $B_{q, p} \equiv\left\{z \in \mathbb{C}, \prod_{k=1}^{q}\left|\left(z-z_{k}\right)\right|^{m_{k}}<r_{2} \prod_{k=q+1}^{p}\left|\left(z-z_{k}\right)\right|^{m_{k}}\right\}$.

These pictures show the topologically different forms of $D_{q, p}$ depending on the relative value of $r_{1}$ and $r_{2}$ when $q=2$ and $p=3$. The pictures are labeled with $\left(r_{1}, r_{2}\right)$. In these pictures $z_{1}\left|z_{1}-z_{2}\right|<\left|z_{1}-z_{3}\right|,\left|z_{2}-z_{3}\right|$ and $r_{2}^{a}>r_{2}^{b}>r_{2}^{c}>r_{2}^{d}$.

where

$$
u \equiv \frac{\prod_{k=1}^{s}\left(z-z_{k}\right) \prod_{k=s+1}^{m}\left(w-z_{k}\right)}{\prod_{k=1}^{s}\left(w-z_{k}\right) \prod_{k=s+1}^{m}\left(z-z_{k}\right)}
$$


and

$$
\begin{aligned}
F_{m}\left(w, z ; z_{1}, \ldots, z_{m}\right) \equiv \frac{1}{w-z}[ & \prod_{k=1}^{s}\left(w-z_{k}\right) \prod_{k=s+1}^{m}\left(z-z_{k}\right) \\
& \left.-\prod_{k=1}^{s}\left(z-z_{k}\right) \prod_{k=s+1}^{m}\left(w-z_{k}\right)\right]
\end{aligned}
$$

into the second. Next we introduce the expansion (2.14) of the factor $(1-u)^{-1}$ in both integrals in (4.10). We observe that $F_{m}\left(w, z ; z_{1}, \ldots, z_{m}\right)$ may be written as

$$
\begin{aligned}
F_{m}\left(w, z ; z_{1}, \ldots, z_{m}\right)= & H_{s}\left(w, z ; z_{1}, \ldots, z_{p}\right) \prod_{k=s+1}^{m}\left(z-z_{k}\right) \\
& -H_{m-s}\left(w, z ; z_{p+1}, \ldots, z_{m}\right) \prod_{k=1}^{s}\left(z-z_{k}\right),
\end{aligned}
$$

where $H_{m}\left(w, z ; z_{1}, \ldots, z_{m}\right)$ is defined in (2.2). Using this decomposition, equation (2.3), and after straightforward calculations we obtain

$$
\begin{aligned}
f(z)= & \sum_{n=0}^{N-1} q_{n, m}(z) \prod_{k=1}^{m}\left(z-z_{k}\right)^{n}+\sum_{n=0}^{N-1} t_{n, m}^{(1)}(z) \frac{\prod_{k=1}^{s}\left(z-z_{k}\right)^{n}}{\prod_{k=s+1}^{m}\left(z-z_{k}\right)^{n}} \\
& +\sum_{n=0}^{N-1} t_{n, m}^{(2)}(z) \frac{\prod_{k=1}^{s}\left(z-z_{k}\right)^{n+1}}{\prod_{k=s+1}^{m}\left(z-z_{k}\right)^{n+1}}+r_{N}(z),
\end{aligned}
$$

where $q_{n, m}(z)$ is given by formulas (2.7) and (2.8) replacing the contour $\mathcal{C}$ by $\Gamma_{1}$. Also,

$$
t_{n, m}^{(1)}(z)=-\sum_{j=1}^{s} b_{n, j} \frac{\prod_{k=1, k \neq j}^{s}\left(z-z_{k}\right)}{\prod_{k=1, k \neq j}^{s}\left(z_{j}-z_{k}\right)}
$$

with

$$
\begin{gathered}
b_{n, j} \equiv \frac{1}{2 \pi i} \int_{\Gamma_{2}} \frac{\prod_{k=s+1}^{m}\left(w-z_{k}\right)^{n}}{\prod_{k=1}^{s}\left(w-z_{k}\right)^{n}} \frac{f(w) d w}{w-z_{j}} \\
t_{n, m}^{(2)}(z)=\sum_{j=s+1}^{m} c_{n, j} \frac{\prod_{k=s+1, k \neq j}^{m}\left(z-z_{k}\right)}{\prod_{k=s+1, k \neq j}^{m}\left(z_{j}-z_{k}\right)}
\end{gathered}
$$

with

$$
c_{n, j} \equiv \frac{1}{2 \pi i} \int_{\Gamma_{2}} \frac{\prod_{k=s+1}^{m}\left(w-z_{k}\right)^{n+1}}{\prod_{k=1}^{s}\left(w-z_{k}\right)^{n+1}} \frac{f(w) d w}{w-z_{j}}
$$

and

$$
\begin{aligned}
r_{N}(z)= & \frac{1}{2 \pi i} \int_{\Gamma_{1}} \frac{f(w) d w}{(w-z) \prod_{k=1}^{m}\left(w-z_{k}\right)^{N}} \prod_{k=1}^{m}\left(z-z_{k}\right)^{N} \\
& -\frac{1}{2 \pi i} \int_{\Gamma_{2}} \frac{\prod_{k=s+1}^{m}\left(w-z_{k}\right)^{N}}{\prod_{k=1}^{s}\left(w-z_{k}\right)^{N}} \frac{f(w) d w}{w-z} \frac{\prod_{k=1}^{s}\left(z-z_{k}\right)^{N}}{\prod_{k=s+1}^{m}\left(z-z_{k}\right)^{N}} .
\end{aligned}
$$


Now we write

$$
\begin{aligned}
& t_{n, m}^{(1)}(z)=-\frac{1}{2 \pi i} \int_{\Gamma_{2}} \frac{\prod_{k=s+1}^{m}\left(w-z_{k}\right)^{n}}{\prod_{k=1}^{s}\left(w-z_{k}\right)^{n}} \sum_{j=1}^{s} \frac{f(w) \prod_{k=1, k \neq j}^{s}\left(z-z_{k}\right)}{\left(w-z_{j}\right) \prod_{k=1, k \neq j}^{s}\left(z_{j}-z_{k}\right)} d w \\
& t_{n, m}^{(2)}(z)=\frac{1}{2 \pi i} \int_{\Gamma_{2}} \frac{\prod_{k=s+1}^{m}\left(w-z_{k}\right)^{n+1}}{\prod_{k=1}^{s}\left(w-z_{k}\right)^{n+1}} \sum_{j=s+1}^{m} \frac{f(w) \prod_{k=s+1, k \neq j}^{m}\left(z-z_{k}\right)}{\left(w-z_{j}\right) \prod_{k=s+1, k \neq j}^{m}\left(z_{j}-z_{k}\right)} d w
\end{aligned}
$$

and repeat the steps following (2.32) in Theorem 2.5 for $q_{n, m}(z), t_{n, m}^{(1)}(z)$ and $t_{n, m}^{(2)}(z)$.

For any $z$ verifying (4.9), we can take simple closed loops $\Gamma_{1}$ and $\Gamma_{2}$ in $\Omega \backslash \Omega_{0}$ such that (see Figure 6(b))

$$
\prod_{k=1}^{p}\left|z-z_{k}\right|^{m_{k}}<\prod_{k=1}^{p}\left|w-z_{k}\right|^{m_{k}} \quad \forall w \in \Gamma_{1}
$$

and

$$
\prod_{k=1}^{q}\left|w-z_{k}\right|^{m_{k}} \prod_{k=q+1}^{p}\left|z-z_{k}\right|^{m_{k}}>\prod_{k=1}^{q}\left|z-z_{k}\right|^{m_{k}} \prod_{k=q+1}^{p}\left|w-z_{k}\right|^{m_{k}}
$$

$\forall w \in \Gamma_{2}$. On these contours $|f(w)|$ is bounded by some constant $C:|f(w)| \leq C$. Introducing these bounds in (4.8) we see that $\lim _{N \rightarrow \infty} r_{N}(z)=0$ and the proof follows.

If the only singularities of $f(z)$ inside $\Omega_{0}$ are just poles at $z_{q+1}, z_{q+2}, \ldots, z_{p}$, then alternative formulas of (4.3), (4.5) and (4.7) for computing the coefficients of the above two-point Taylor-Laurent expansion is given in the following proposition.

Proposition 4.2. Suppose that $g_{k}(z) \equiv\left(z-z_{k}\right)^{\rho_{k}} f(z)$ is an analytic function in $\Omega$ for certain $\rho_{k} \in \mathbb{N}$ and $k=q+1, q+2, \ldots, p$. Define $g_{k}(w)=f(w)$ for $k=1,2,3, \ldots, q$. Then the coefficients $a_{n, j, l}, b_{n, j, l}$ and $c_{n, j, l}$ in the expansion (44.1) are also given by the formulas:

$$
\begin{aligned}
a_{n, j, l}= & \left.\left.\sum_{k=1, k \neq j}^{q} D_{w}^{n m_{k}-1}\left[\frac{f(w)}{\left(w-z_{j}\right)^{l+1} \prod_{s=1, s \neq k}^{p}\left(w-z_{s}\right)^{n m_{s}}}\right]\right|_{w=z_{k}}\right]\left.\left.\right|_{k=q+1, k \neq j} D_{w}^{n m_{k}+\rho_{k}-1}\left[\frac{g_{k}(w)}{\left(w-z_{j}\right)^{l+1} \prod_{s=1, s \neq k}^{p}\left(w-z_{s}\right)^{n m_{s}}}\right]\right|_{w=z_{k}} \\
& \left.+\sum_{w}^{n}\right]\left.\right|_{w=z_{j}}, \\
& +D_{w, j}^{n m_{j}+\rho_{j}+l}\left[\frac{g_{j}(w)}{\prod_{s=1, s \neq j}^{p}\left(w-z_{s}\right)^{n m_{s}}}\right. \\
b_{n, j, l} & =\left.\sum_{k=q+1}^{p} D_{w}^{\rho_{k}-n m_{k}-1}\left[\frac{g_{k}(w) \prod_{s=q+1, s \neq k}^{p}\left(w-z_{s}\right)^{n m_{s}}}{\left(w-z_{j}\right)^{l+1} \prod_{s=1}^{q}\left(w-z_{s}\right)^{n m_{s}}}\right]\right|_{w=z_{k}},
\end{aligned}
$$




$$
\begin{aligned}
c_{n, j, l}= & \left.\sum_{k=q+1, k \neq j}^{p} D_{w}^{\rho_{k}-(n+1) m_{k}-1}\left[\frac{g_{k}(w) \prod_{s=q+1, s \neq k}^{p}\left(w-z_{s}\right)^{(n+1) m_{s}}}{\left(w-z_{j}\right)^{l+1} \prod_{s=1}^{q}\left(w-z_{s}\right)^{(n+1) m_{s}}}\right]\right|_{w=z_{k}} \\
& +\left.D_{w}^{\rho_{j}-(n+1) m_{j}+l}\left[\frac{g_{j}(w) \prod_{s=q+1, s \neq j}^{p}\left(w-z_{s}\right)^{(n+1) m_{s}}}{\prod_{s=1}^{q}\left(w-z_{s}\right)^{(n+1) m_{s}}}\right]\right|_{w=z_{j}} .
\end{aligned}
$$

Proof. We deform both the contour $\Gamma_{1}$ in equation (4.3) and the contour $\Gamma_{2}$ in equations (4.5) and (4.7) into any contour of the form $\mathcal{C}_{1} \cup \mathcal{C}_{2} \cup \cdots \cup \mathcal{C}_{p}$ contained in $\Omega$, where $\mathcal{C}_{k}, k=1,2, \ldots, p$ is a simple closed loop which encircles the point $z_{k}$ in the counterclockwise direction with $z_{j}$ not inside $\mathcal{C}_{k}, j=1,2, \ldots, p, j \neq k$ (see Figure 3 (c)). Then,

$$
\begin{aligned}
c_{n, j, l}= & \sum_{k=q+1, k \neq j}^{p} \frac{1}{2 \pi i} \int_{\mathcal{C}_{k}} \frac{\prod_{s=q+1, s \neq k}^{p}\left(w-z_{s}\right)^{(n+1) m_{s}}}{\left(w-z_{j}\right)^{l+1} \prod_{s=1}^{q}\left(w-z_{s}\right)^{(n+1) m_{s}}} \frac{g_{k}(w) d w}{\left(w-z_{k}\right)^{\rho_{k}-(n+1) m_{k}}} \\
& +\frac{1}{2 \pi i} \int_{\mathcal{C}_{j}} \frac{\prod_{s=q+1, s \neq j}^{p}\left(w-z_{s}\right)^{(n+1) m_{s}}}{\prod_{s=1}^{q}\left(w-z_{s}\right)^{(n+1) m_{s}}} \frac{g_{j}(w) d w}{\left(w-z_{j}\right)^{\rho_{j}-(n+1) m_{j}+l+1}} .
\end{aligned}
$$

From this, equations (4.24), (4.25) and (4.26) follow.

Remark 4.3. Let $z$ be a real or complex variable and suppose that $\left(z-z_{k}\right)^{\rho_{k}} f(z)$ is $\rho_{k}-1$ times differentiable at $z_{k}$ for certain $\rho_{k} \in \mathbb{N}$. Define

$g(z) \equiv f(z)-\sum_{n=0}^{M} t_{n, m}^{(1)}(z) \frac{\prod_{k=1}^{q}\left(z-z_{k}\right)^{n m_{k}}}{\prod_{k=q+1}^{p}\left(z-z_{k}\right)^{n m_{k}}}-\sum_{n=0}^{M} t_{n, m}^{(2)}(z) \frac{\prod_{k=1}^{q}\left(z-z_{k}\right)^{(n+1) m_{k}}}{\prod_{k=q+1}^{p}\left(z-z_{k}\right)^{(n+1) m_{k}}}$,

where $M \equiv\left\lfloor\operatorname{Max}\left\{\left(\rho_{q+1}-1\right) / m_{q+1},\left(\rho_{q+2}-1\right) / m_{q+2}, \ldots,\left(\rho_{p}-1\right) / m_{p}\right\}\right\rfloor$ and $t_{n, m}^{(1)}(z)$ and $t_{n, m}^{(2)}(z)$ are the polynomials defined in (4.4), (4.6), (4.25) and (4.26). Then, the thesis of Proposition 2.9 holds for $f(z)$ replaced by $g(z)$.

Moreover, if $\prod_{k=q+1}^{p}\left(z-z_{k}\right)^{\rho_{k}} f(z)$ is an analytic function in $\Omega$, then the thesis of Theorem 2.5 applies to $g(z)$.

\section{Discussion AND CONCLUDing REMARKS}

In an earlier paper [6] we have discussed the theory of two-point Taylor expansions, two-point Laurent expansions and two-point Taylor-Laurent expansions. In the present paper we have generalized these two-point cases to multi-point cases. We have given details on the regions of convergence and on representations of the coefficients and the remainders of the expansions in terms of Cauchy-type integrals. 
Multi-point Taylor expansions are related with topics from interpolation theory, in particular with the Newton interpolation theory with applications in numerical analysis. For example, applications can be found in initial and boundary value problems in connection with ordinary differential equations and in numerical quadrature of integrals.

From the point of view of interpolation theory detailed information on multipoint expansions can be found in $[8$, Chapters 3 and 8 . The theory of several-point Taylor expansions is discussed in Chapter 3 of [8], although in a setting that is different from our approach. Our approach gives explicit Cauchy-type integrals of coefficients and remainders which cannot be found in Walsh's approach. In particular, we cannot find explicit formulas for the polynomials $q_{n, m}(z)$ of formula (2.27) as we have in (2.28)- 2.29). Knowledge of these explicit formulas is necessary to construct asymptotic expansions of integrals with several saddle points.

In addition to this, our Laurent and Taylor-Laurent expansions are new. They have a formal similarity with the rational approximations of Chapter 8 of $[8]$ : they involve negative powers of $z$. But they are completely different. The rational approximations, in particular the Padé-type approximations $P_{n}(z) / Q_{m}(z)$, are of interpolatory type. These are generalizations of the Taylor polynomial at several points: a quotient of polynomials instead of a polynomial. However, our expansions (3.1) or (4.1) have a different form and a different approximation property: they approach not only at regular points like Padé-type approximations but also at singular points of $f(z)$. And of course, the regions and convergence properties in 8] are different from ours.

Apart from applying the present results in problems from interpolation theory, in particular in problems from numerical analysis, we expect to find applications in asymptotic analysis of integrals, which application area is our main motivation; see [6]. In that paper certain orthogonal polynomials have been considered and we have given new convergent expansions that also have an asymptotic property for large values of a parameter (the degree $n$ of the polynomials). Orthogonal polynomials and special functions can be studied when the variable and several parameters are large. In that case more than one or two so-called critical points occur that may give the main contributions to the integral, and expansions of analytic functions at these points again give the possibility of constructing new convergent expansions with an asymptotic property. This method avoids the complicated conformal mapping of the phase function of the integral into a standard form (say a cubic or higher polynomial). In addition, when the critical points are multiple poles, Laurenttype expansions may be considered. A few application areas are mentioned in the Introduction (see the integral in (1.4)), which we expect to approximate in terms of Airy functions and the Pearcey integral (1.5) and its derivative with respect to $x$ and $y$.

\section{REFERENCES}

1. C. Chester, B. Friedman, and F. Ursell, An extension of the method of steepest descent. Proc. Cambridge Philos. Soc. 53 (1957), 599-611. MR 19:853a

2. K.A. Driver and N.M. Temme, On polynomials related with Hermite-Padé approximations to the exponential function. J. Approx. Theory. 65 (1998), 101-122. MR 99j:41028

3. A.J.E.M. Janssen, On the asymptotics of some Pearcey-type integrals. J. Phys. A: Math. Gen. 25 (1992), L823-L831. MR 93f:41047 
4. N.P. Kirk, J.N.L. Connor, P.R. Curtis and C.A. Hobbs, Theory of axially symmetric cusped focusing: numerical evaluation of a Bessoid integral by an adaptive contour algorithm. J. Phys. A: Math. Gen. 33 (2000), 4797-4808. MR 2001h:78004

5. José L. Lopez and Nico M. Temme, Asymptotic expansions of Charlier, Laguerre and Jacobi polynomials. Accepted for publication in The Proceedings of the Royal Society of Edinburgh A (Mathematics).

6. José L. Lopez and Nico M. Temme, Two-point Taylor expansions of analytic functions. Stud. Appl. Math. 109 (2002), 297-311. MR 2003g:30005

7. Raimundas Vidunas and Nico M. Temme, Symbolic evaluation of coefficients in Airy-type asymptotic expansions. J. Math. Anal. Appl. 269 (2002), 317-331. MR 2003c:41041]

8. J. L. Walsh, Interpolation and Approximation by rational functions in the complex domain, Amer. Math. Soc., Providence, RI, 1969. MR 36:1672a

9. R. Wong, Asymptotic Approximations of Integrals, Academic Press, New York, 1989. MR 90j:41061

Departamento de Matématica e Informática, Universidad Pública de Navarra, 31006PAMPlona, Spain

E-mail address: j1.lopez@unavarra.es

CWI, P.O. Box 94079, 1090 GB Amsterdam, The Netherlands

E-mail address: nicot@cwi.nl 